\title{
Comparing the Effectiveness of Health Program in Thailand and Japan
}

Suchinda Maruo Jarupat ${ }^{1}$, Wanna Sanongdetch ${ }^{1}$, Supalax Choeychom ${ }^{1}$, Sirintorn Chansirikarn ${ }^{1}$, Supunnee Thrakul ${ }^{1}$, Rutja Phuphaibul ${ }^{1}$, Sampun Phuphaibul $^{2}$, Saiki Terasawa ${ }^{3}$, Keisuke Nakade $^{3}$, Toshiaki Watanabe ${ }^{3}$, Yuki Murata ${ }^{3}$ and Koji Terasawa ${ }^{3^{*}}$

${ }^{1}$ Ramathibodi School of Nursing, Faculty of Medicine, Ramathibodi Hospital, Mahidol University, Bangkok, Thailand

${ }^{2}$ Leader of Ban Salawan health promoting hospital elderly club, Salaya Sub-district, Nakornprathom, Thailand

${ }^{3}$ Shinshu University, Naganoshi, Nagano 380-0928, Japan

*Corresponding author: Koji Terasawa, Graduate School of Medicine, Shinshu University, 3-1-1 Asahi, Matsumoto, Nagano 390-8621 Japan, Tel: +81-26-238-4213, Email:kterasa@shinshu-u.ac.jp

Received date: Aug 05, 2015, Accepted date: Sep 07, 2015, Published date: Sep 15, 2015

Copyright: ( 2015 Jarupat SM et al. This is an open-access article distributed under the terms of the Creative Commons Attribution License, which permits unrestricted use, distribution, and reproduction in any medium, provided the original author and source are credited.

\begin{abstract}
Objective: This study aimed to establish appropriate health education in Thailand to compare the health education of Thailand and Japan.

Method: Participants in Salaya, Thailand, totalled of 24 people aged $69.1 \pm 6.6$ years, whereas participants in Minowa, Japan totalled 46 people aged $62.7 \pm 4.7$ years. Implementing the health program lasted 6 months in Salaya and 10 months in Minowa. We measured the energy expenditure using a pedometer and implementing Go/No-Go task for the brain function test and physical fitness tests in the before and after this period.

Results: The results of Salaya showed the average walking steps were 4,012. As for Minowa, the average walking steps were 8,644. In Minowa, the Go/No-Go task number of error responses significantly decreased after the program, although Salaya was not significantly different. As for Salaya, the results for grip strength and sit ups significantly decreased after the program, whereas 6-minute walk significantly improved. In Minowa, the results of the handgrip strength, sit ups, sit-and-reach flexibility, 10-meter obstacle walk, and the 6-minute walk significantly improved after the program. The handgrip strength and sit ups of Minowa showed a significant difference from those of Salaya.
\end{abstract}

Conclusion: By doing the 90-minute strength and weight training once a week in Minowa the participants may have encouraged one another to a superior number of walking steps, and better rest results on the Go/No-Go task and the physical fitness tests compared with those of Salaya.

Keywords: Health education; Pedometers; Go/No-Go task; Physical fitness

\section{Introduction}

A Canadian politician Marc Lalonde declared that the focus of public health was going to be shifted from disease prevention to health promotion in 1974 [1]. A new national policy for health promotion, catch-phrased "Healthy People", was enacted to change the core philosophy of medicine from advanced treatment to primary health care, with more emphasis on prevention. In 1986, the American government designated a city government which contributed to health promotion in a local area, whose name was called "healthy city" [2]. The Japanese government also enacted a national policy, "Kenkko Nippon 21" with more focus on the decrease in death rate for the middle-aged, the extension of life span, the improvement of quality of life, and primary care and prevention [3]. In 2002, the Japanese government enacted a developed version of the health-promotion law, which imposes demand for a health-promotion policy on each prefecture and a conscious effort for the local policy on every city. The Japanese Ministry of Health, Labour and Welfare introduced an idea for a national policy to improve the prevention and treatment for metabolic syndrome, which will plan to start charging a penalty to the National Federation of Health Insurance Societies in 2013 in case residents do not meet preventive criteria for the syndrome. This economic pressure seems to have encouraged the practice of health education at a national, state, and local city level [4].

An active health program, developed by the Japanese authors, is implemented by measuring energy expenditure and conducting the blood test, the brain function test, and the physical fitness test as well as providing educational seminars regarding exercise and nutrition, and recreational activities such as hiking and cooking. The practice of health education in Thailand usually includes monthly meetings with club members, periodic seminars to develop participants' leadership, with Yoga and stretch exercise as well; however, a lack of systematic evaluation after a program seems to make it difficult for Thai's health practitioners to decide the effectiveness of a health program.

This study aimed to establish appropriate health education in Thailand to compare the health education of Thailand and Japan.

In particular, it can be beneficial to share the health promotion in Minowa with other cities and countries because Nagano prefecture including Minowa has been one of the national longevity areas over the last decades. Since Japan was the world's highest longevity country in 2005, Minowa is expected to be one of the world-class areas where the longevity is foreseen. 


\section{Method}

\section{General methods}

The authors implemented a 6-month health program from Sep 2011 to Feb 2012 in the district of Salaya in Bangkok. This program was the latest version developed by the authors in Japan, which measures the energy expenditure by pedometers, and tests the brain function and physical fitness. The authors originally planned to practice the health program once a month in Salaya, but the only four times program was possible due to the flood that damaged the district from Nov 2011 to Feb. The participants in Salaya performed the following recreational activities: modified Boonmee long stick exercise on balance, health education on blood pressure, Thai Yoga and walking exercise (Table 1). In Salaya, 72 elderly aged $67.8 \pm 8.0$ years (Mean \pm SD) initially participated in the program, but only 7 men aged $69.9 \pm 5.6$ years and 17 women aged $69.6 \pm 6.6$ years, for a total of 24 people aged $69.1 \pm 6.6$ years completed the program due to the flood. The 10-month health program was concurrently implemented in Minowa, Japan from May 2011 to February 2012. In Minowa, a total of 46 people aged $62.7 \pm 4.7$ years participated in the program. However the persons who participated till the last were the 20 elderly men aged $64.3 \pm 4.5$ years and 24 elderly women aged $60.8 \pm 3.9$ years, total 44 aged $62.3 \pm 4.5$ years. The participants in Minowa also took a series of recreational activities: walking exercise, Tai Chi Chuan, nature tour, cooking practice, medical seminars on blood pressure, mouth care, nutrition balance and performed 90-minute strength and weight training once a week (Table 1).

The latest guideline of the Helsinki Declaration was adopted by the Institutional Ethics Committee of Mahidol University(Mahidol Univ. IRB: 01-58-10) and Shinshu University (UMIN000009309). Written informed consents were obtained from Salaya and Minowa elderly people.

\begin{tabular}{|c|c|c|}
\hline Month & Salaya program & Minowa program \\
\hline 5 & & $\begin{array}{l}\text { The measurement before the health education, distribution of the pedometer and training once a } \\
\text { week }\end{array}$ \\
\hline 6 & & Lecture on the importance of health and training once a week \\
\hline 7 & & Practical recreation and training once a week \\
\hline 8 & $\begin{array}{l}\text { The measurement before the health } \\
\text { education, distribution of the pedometer }\end{array}$ & Hiking and training once a week \\
\hline 9 & $\begin{array}{l}\text { Cannot carry out the program because of } \\
\text { floods }\end{array}$ & Lcture on nutrition and training once a week \\
\hline 10 & $\begin{array}{l}\text { Cannot carry out the program because of } \\
\text { floods }\end{array}$ & Practical walking and training once a week \\
\hline 11 & $\begin{array}{l}\text { Modified Boonmee long stick exercise on } \\
\text { balance }\end{array}$ & Lecture on blood pressure and training once a week \\
\hline 12 & Health education on blood pressure & Lecture on dental health and training once a week \\
\hline 1 & Thai Yoga & Tai Chi Chuan and training once a week \\
\hline 2 & $\begin{array}{l}\text { Walking exercise and the measurement after } \\
\text { health education }\end{array}$ & The measurement after health education and training once a week \\
\hline
\end{tabular}

Table 1: Program contents of the health education in Salaya and Minowa

\section{Pedometer}

The number of walking steps and the amount of energy expenditure were measured during the Aug 2011 to Feb 2012 in the Salaya and May 2011 to February 2012 in Minowa. The weight Bering Index (WBI) gave the approximate number of daily walking steps [5]. A recent model (Acos Inc., FS50) enabled it to transfer the saved data from the pedometer to a personal computer. The pedometer measured the number of walking steps when a participant walked more than 4 METS. Participants reported their results to a project leader when participants gathered for a monthly meeting.

\section{The Go/No-Go task}

The Go/No-Go task [6] was used in order to estimate as a battery test the inhibitory decision process [7] and dementia process [8] in prefrontal cortex, whose task consisted of three experimental stages: formation, differentiation, and reverse differentiation. First, in the formation stage, participants were instructed to squeeze a rubber ball in response to a red light. Second, during the differentiation stage, they squeezed a rubber ball in response to a red light, but not a yellow light, when a red or yellow light was randomly displayed. Third, during the reverse differentiation stage, participants squeezed a rubber ball in response to a yellow light but not a red light. In each of the differentiation and reverse differentiation stages participants completed 20 trials. Red and yellow lights were equally likely to be displayed 10 times each. In this paper, the term "forget" indicates an incorrect response when participants did not squeeze a rubber ball when it is to be squeezed. On the contrary, the term "mistake" means an incorrect response when participants did squeeze a rubber ball when it was not supposed to be squeezed.

Physical test

The physical tests approved by the Japanese Ministry of Education, Culture, Sports, Science and Technology [9] were composed of six physical assessments: grip strength, sit ups, sit-and-reach flexibility, 
Citation: Maruo SJ, Sanongdetch W, Choeychom S, Chansirikarn S, Thrakul S, et al. (2015) Comparing the Effectiveness of Health Program in Thailand and Japan. J Nurs Care 4: 298. doi:10.4172/2167-1168.1000298

Page 3 of 6

single leg stance, 10-meter obstacle walk and a 6-minute walk. The participant's physical ability was assessed before and after the health program.

Statistical Analysis

The paired-t test was used to compare the results before and after participating in the health program. Another factor of place (Salaya vs. Minowa) was analyzed by Two- way repeated measure ANOVA. The walking steps for these two places were compared by the independent t-test. The 18th version of SPSS was used for the analysis.

\section{Result}

\section{The number of walking and exercise steps}

The subjects were 24 elderly people aged $69.1 \pm 6.6$ years in Salaya and 44 elderly people aged $62.3 \pm 4.5$ years in Minowa. Figure 1 showed the average walking steps and exercise steps in each month when the speed was defined as more than 4 METS. In Minowa, the average walking steps and exercise steps gradually increased from May to October. Walking steps and exercise steps of October showed $9382.6 \pm$ 512.6 and 6141.7 \pm 467.0, respectively. However, both walking and exercise steps decreased from November to February. Walking steps and exercise steps of February showed $7991.4 \pm 517.1$ and 5515.7 \pm 489.3, respectively. In Salaya, the average walking steps and exercise steps in August were fewer than those in the other months. Walking steps and exercise steps of August showed 1460.1 \pm 233.1 and 640.4 \pm
84.2. However, those numbers of steps increased after October to $4993.7 \pm 175.6$ and $2140.1 \pm 90.7$ (Figure 1).

As shown in Table 2, the Salaya results showed that the mean walking steps were $4011.7 \pm 190.1$, whereas the mean exercise steps were 1700.9 \pm 96.4. The Minowa results showed that the mean walking steps were $8648.0 \pm 165.7$ whereas the mean exercise steps were $5482.3 \pm 148.3$. In Minowa, the average walking steps $(\mathrm{t}=11.8, \mathrm{p}>0.000)$ and exercise steps $(\mathrm{t}=-3.9, \mathrm{p}>0.002)$ showed significant increase, compared with those of Salaya (Table 2).

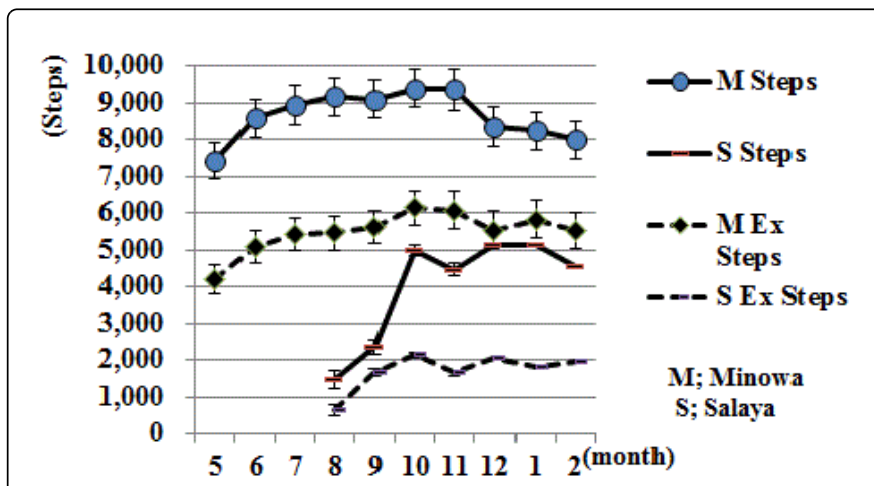

Figure 1: Walking steps and exercise steps

\begin{tabular}{|c|c|c|c|c|c|c|c|c|}
\hline & & \multicolumn{2}{|l|}{ Salaya } & \multirow{2}{*}{$\begin{array}{l}\text { paired } \\
\text { p-value }\end{array}$} & \multicolumn{2}{|l|}{ Minowa } & \multirow{2}{*}{\begin{tabular}{|l|} 
paired \\
p-value
\end{tabular}} & \multirow{2}{*}{$\begin{array}{l}2 \text { way } \\
\text { ANOVA } \\
\text { p-value }\end{array}$} \\
\hline & & Before & After & & Before & After & & \\
\hline \multicolumn{9}{|c|}{$\begin{array}{l}\text { Response time of Go/No-Go task } \\
\text { (ms) }\end{array}$} \\
\hline Formation & & $295.1 \pm 18.3$ & $341.2 \pm 29.8$ & NS & 256.6. \pm 8.4 & $244.1 \pm 5.5$ & NS & * \\
\hline Differentiation & & $442.5 \pm 23.8$ & $399.3 \pm 12.9$ & NS & $403.1 \pm 8.7$ & $382.1 \pm 9.4$ & NS & NS \\
\hline Reverse Differentation & & $432.5 \pm 16.2$ & $410.6 \pm 14.9$ & NS & $430.3 \pm 10.4$ & $414.5 \pm 11.0$ & NS & NS \\
\hline Total & & $390.1 \pm 14.6$ & $383.7 \pm 14.4$ & NS & $387.3 \pm 7.6$ & $368.9 \pm 8.3$ & * & NS \\
\hline \multicolumn{9}{|c|}{$\begin{array}{l}\text { The number of error response } \\
\text { (times) }\end{array}$} \\
\hline \multirow[t]{2}{*}{ Differentiation } & Forget & $0.6 \pm 0.3$ & $0.2 \pm 0.1$ & NS & $0.1 \pm 0.0$ & $0.1 \pm 0.1$ & NS & NS \\
\hline & Mistake & $3.7 \pm 0.5$ & $3.0 \pm 0.4$ & NS & $2.2 \pm 0.3$ & $1.3 \pm 0.2$ & $* * *$ & NS \\
\hline \multirow[t]{2}{*}{ Reverse Differentation } & Forget & $0.9 \pm 0.2$ & $0.6 \pm 0.2$ & NS & $0.1 \pm 0.0$ & $0.2 \pm 0.2$ & NS & NS \\
\hline & Mistake & $3.2 \pm 0.3$ & $2.6 \pm 0.4$ & NS & $0.9 \pm 0.2$ & $0.6 \pm 0.2$ & NS & NS \\
\hline \multirow[t]{3}{*}{ Total } & Forget & $1.5 \pm 0.4$ & $0.8 \pm 0.2$ & NS & $0.1 \pm 0.0$ & $0.2 \pm 0.2$ & NS & NS \\
\hline & Mistake & $6.9 \pm 0.7$ & $5.6 \pm 0.8$ & NS & $3.0 \pm 0.4$ & $1.9 \pm 0.3$ & $* * *$ & NS \\
\hline & Both & $8.4 \pm 0.9$ & $6.5 \pm 0.9$ & NS & $3.1 \pm 0.4$ & $2.1 \pm 0.4$ & * & NS \\
\hline \multicolumn{9}{|l|}{ Physical fitness test } \\
\hline Handgrip strength $(\mathrm{kg})$ & & $22.6 \pm 1.7$ & $20.4 \pm 1.5$ & * & $33.6 \pm 1.5$ & $37.3 \pm 1.5$ & $* \star *$ & NS \\
\hline Sit-up (times) & & $4.1 \pm 1.0$ & $1.8 \pm 0.9$ & * & $11.9 \pm 0.9$ & $15.3 \pm 0.9$ & $* * *$ & $* *$ \\
\hline
\end{tabular}


Page 4 of 6

\begin{tabular}{|l|l|l|l|l|l|l|l|l|}
\hline Sit and reach flexibility (cm) & & $31.2 \pm 1.9$ & $33.3 \pm 2.0$ & NS & $42.6 \pm 1.3$ & $47.4 \pm 1.1$ & $* * *$ & NS \\
\hline Eyes open single leg stance (sec) & & $22.6 \pm 6.2$ & $25.2 \pm 8.6$ & NS & $106.0 \pm 4.5$ & $105.7 \pm 4.5$ & NS & NS \\
\hline 10-meter obstacle (sec) & & $11.5 \pm 1.0$ & $9.7 \pm 0.3$ & NS & $6.8 \pm 0.1$ & $5.1 \pm 0.1$ & $* * *$ & NS \\
\hline 6-minute walk (m) & & $454.3 \pm 11.5$ & $493.5 \pm 14.9$ & $* *$ & $622.2 \pm 7.3$ & $719.9 \pm 9.2$ & $* * *$ & $* *$ \\
\hline Walking & & & & & & & & \\
\hline Walking/day (steps) & & $4011.7 \pm 190.1$ & & & $8648.0 \pm 165.7$ & & $* * *$ & \\
\hline Exercise/day (steps) & & $1700.9 \pm 96.4$ & & & $5482.3 \pm 148.3$ & & $* *$ & \\
\hline
\end{tabular}

Table 2: Results before and after the health program in Salaya and Minowa, (Mean \pm SE, NS: not significant, : $\mathrm{p}<0.05,: \mathrm{p}<0.01,: \mathrm{p}<0.001$ )

\section{Go/No-Go tasks}

In Minowa, the reaction time became significantly shorter in total $(\mathrm{t}$ $=2.3, \mathrm{p}<0.05)$ after the health program. As for the number of errors for Salaya, there were no significant differences before and after the health program in the three stages of experiment, although in Minowa, the number of errors for mistake of differentiation $(\mathrm{t}=4.1, \mathrm{p}<0.000)$, total of mistake $(t=3.7, p<0.001)$ and both of total forgot and mistake $(\mathrm{t}=2.5, \mathrm{p}<0.016)$ was statistically significance. The Go/No-go task results were compared between the before and after program for Salaya and Minowa. The reaction time for the formation stage $(\mathrm{F}=-4.0, \mathrm{p}<$ 0.05) was significantly longer in Salaya than Minowa. As for the number of errors, there was no significant difference between Salaya and Minowa (Table 1).

\section{Physical strength test}

The Salaya showed that the grip strength $(t=2.2, \mathrm{p}<0.043)$ and situps $(t=2.1, p<0.049)$ significantly decreased after the participants joined the health program. In contrast, the distance of 6-minute-walk $(t=-3.2, p<0.005)$ significantly increased after the program. As for the result of Minowa, the grip strength $(\mathrm{t}=-7.4, \mathrm{p}<0.000)$, sit ups $(\mathrm{t}=$ -6.7, $\mathrm{p}<0.000)$, sit-and-reach flexibility $(\mathrm{t}=-8.6, \mathrm{p}<0.000), 10$-meter obstacle walk $(\mathrm{t}=17.3, \mathrm{p}<0.000)$ and 6 -minute walk $(\mathrm{t}=-13.4, \mathrm{p}<$ $0.000)$ significantly improved performance after the program. The sit ups $(\mathrm{F}=7.8, \mathrm{p}<0.01)$ and 6 -minute walk $(\mathrm{F}=7.6, \mathrm{p}<0.001)$ of Minowa showed a significant difference than those of Salaya (Table 1).

\section{Discussion}

\section{The number of walking steps during the health program}

During the health program, a few studies showed that participants with pedometers walked for a longer distance for a longer time than those without pedometers $[10,11]$. For instance, participants wearing pedometers showed significantly longer walking time than those without pedometers [12]. In this study, wearing pedometers might have also helped to increase their walking distance and time. From a perspective of health education, it is important to encourage participants to increase the quality and quantity of exercise [13]. An additional, educational intervention is necessary to improve the quality of future programs.

The average walking steps and exercise steps of Minowa gradually increased from May to October, whereas those averages decreased from November to February. This decreased number of walking steps is probably due to the difficulty of walking in the cold, snowy winter in
Minowa. On the other hand, in Salaya, the average walking steps and exercise steps in August were fewer than those in other months. However, the numbers of walking steps returned to 4000 to 5000 steps after October. This temporary decrease of walking steps in August is probably because they were unable to walk outside due to the flood that devastated the region. It is unclear why the walking steps in Salaya did not increase more than 4000 to 5000 steps even though the walking steps in Minowa consistently 8000 to 9000 steps. Even if the flood prevented people in Salaya from walking outside, the number of walking steps may suggest their daily average number of walking steps. We need to determine their average walking steps when it is not affected by the flood. On the other hand, this result will become the precious document of the Thai population at the time of the flood.

\section{Physiological and behavioral studies inferred from the Go/No-Go task}

The origin of Go/No-Go task presumably dates back to the Pavlov's well-known conditioning experiment which uses the sound of bells, whose concept was developed into the stimulus of light. The dysfunction of the prefrontal cortex is presumably associated with the conditioning response [14].

Sasaki et al [15] reported No-Go potential appears when it is not allowed to grasp a rubber ball, was found from monkeys and humans in the lateral prefrontal cortex. This outcome seems to be related to the area of 46 which plays a crucial role in working memory [16].

We used the go/no-go task as investigation about the development of the brain function of the child [7]. It is reported that go/no-go task could apply to investigation of the brain function for screening test of the dementia [8]. The previous study [17-19] of the Go/No-Go experiment reported that the reaction time became faster and the number of error responses became fewer when participants continue to exercise for 10 months with the average of 7,000 steps a day. The two-year continuation of this walking exercise seems to speed up the reaction time and decrease the number of error responses. The results of Salaya and Minowa showed that the average reaction time of the Go/No-Go task became faster after the program. As for the number of errors for Salaya, there were no significant differences before and after the health program, although in Minowa, the number of errors for mistake of differentiation, total of mistake and both of totals forgot and mistake decrease was statistical significance. These results suggest that in Minowa the brain function including working memory is better than Salaya after the program. 


\section{Physical fitness test}

The practice of physical measurement differs from country to country. There are two types of exercise programs in Japan. One $[4,20]$ comprises six physical tests to increase functionality of the motor system: hand grip strength, muscle strength in the lower extremities, functional reach test, eyes-open single leg stance, timed up \& go test, $5 \mathrm{~m}$-normal-and-maximal walk. This Physical fitness test for the care for the aged is recommended by the Ministry of Health, Labour and Welfare. The other $[7,21]$ comprises six physical tests designed for healthy elderly people is recommended by the Ministry of Education, Culture, Sports, Science and Technology : hand grip strength, sit ups, sit-and-reach flexibility, single leg stance, 10-meter obstacle walk and 6-minute walk. We have chosen this Physical fitness test for healthy elderly people.

In Minowa, the five physical fitness tests significantly improved after the program. The results of Salaya showed that the grip strength and sit-ups significantly decreased after the participants joined the health program. In contrast, the distance of a 6-minute-walk significantly increased after the program. The smaller increments of improvement in Salaya may be explained by several reasons. One is the two-month flood that devastated the region, which made participants unable to perform the amount of exercise. The results of Salaya, however, showed that the 6-minute-walk improved after the program. This improvement may be due to the procedural difference of the 6-minute-walk; that is, it was measured in an indoor, small gym before the program, but was measured in a bigger, spacious gym after the program. Using similar facilities for implementing these physical fitness tests would be important for future studies. The sit ups and 6-minute-walk tests of Minowa showed significant differences from those of Salaya. Reflection on some of these may make it clear that the muscle weakness of the Salaya people was caused by the two-month flood that devastated the region.

The superior number of walking steps in Minowa may reflect better performance of the Go/No-Go task as well as better results on the physical fitness tests compared with those of Salaya. The flooding in Salaya might have been a factor in decreasing the improvement of test results.

\section{Conclusion}

This study aimed to establish a health program in Thailand and assess the effectiveness of both Thailand and Japan. Participants in Salaya, Thailand, were a total of 24 people aged $69.1 \pm 6.6$ years, whereas participants in Minowa, Japan were a total of 46 people aged $62.7 \pm 4.7$ years. Implementing the health program lasted 6 months in Salaya and 10 months in Minowa.

The average walking steps and exercise steps of Minowa gradually increased from May to October, whereas those averages decreased from November to February. On the other hand, in Salaya, the average walking steps and exercise steps in August were fewer than those in other months. However, the numbers of walking steps returned to 4000 to 5000 steps after October.

The results of Salaya and Minowa showed that the average reaction time of the Go/No-Go task became faster after the program. As for the number of errors for Salaya, there were no significant differences before and after the health program, although in Minowa, the number of errors for mistake of differentiation, total of mistake and both of total forgot and mistake decrease was statistical significance. These results suggest that in Minowa the brain function including working memory is better than Salaya after the program.
In Minowa, the five physical fitness tests significantly improved after the program. The results of Salaya showed that the grip strength and sit-ups significantly decreased after the participants joined the health program. In contrast, the distance of a 6-minute-walk significantly increased after the program. The smaller increments of improvement in Salaya may be explained by several reasons. One is the two-month flood that devastated the region, which made participants unable to perform the amount of exercise. The results of Salaya, however, showed that the 6-minute-walk improved after the program. This improvement may be due to the procedural difference of the 6-minute-walk; that is, it was measured in an indoor, small gym before the program, but was measured in a bigger, spacious gym after the program. Using similar facilities for implementing these physical fitness tests would be important for future studies.

The superior number of walking steps in Minowa may reflect better performance of the Go/No-Go task as well as better results on the physical fitness tests compared with those of Salaya. The flooding in Salaya might have been a factor in decreasing the improvement of test results.

\section{Acknowledgments}

Suchinda Jarupat Maruo was supported by the Development potentials of Thai people project, Faculty of Medicine, Ramathibodi Hospital, Mahidol University. Koji Terasawa was supported by a Grantin-Aid for the Scientist of the Ministry of Education, Culture, Sports, Science and Technology of Japan (Houga: 23650426, KibanA: 25257101).

\section{References}

1. McGinnis JM, Girasek DC, Wypijewski C (1992) Objectives for the year 2000. Clin Chem 38: 1552-1554.

2. Kickbusch I (1986) Health promotion: a global perspective. Can J Public Health 77: 321-326.

3. Japan health promotion \& foundation 2000: http:// www.kenkounippon21.gr.jp/

4. Yokoe M, Takada T, Mayumi T, Yoshida M, Isaji S5, et al. (2015) Japanese guidelines for the management of acute pancreatitis: Japanese Guidelines 2015. J Hepatobiliary Pancreat Sci 22: 405-432.

5. Kikawa A, Yamamoto $\mathrm{T}$ (1991) The functional muscular strength measurement. Rating system of Weight Bearing Index. The Japanese Orthopaedic Society for Sports Medicine 463-468.

6. Masaki T, Moriyama G (1971) Study on types of human higher nervous activity. Tokyo University of Science press 4,pp 69-81.

7. Terasawa K, Tabuchi H1, Yanagisawa H2, Yanagisawa A3, Shinohara K4, et al. (2014) Comparative survey of go/no-go results to identify the inhibitory control ability change of Japanese children. Biopsychosoc Med 8: 14 .

8. Terasawa K, Misaki S, Murata Y, Watanabe T, Terasawa S, Kobayashi T, Yong Z, Nakajima K, Suchinda M, Nakade K. (2014) Relevance between Alzheimer's disease patients and normal subjects using go/no-go tasks and Alzheimer assessment scores: Journal of Child and Adolescent Behavior 1-5.

9. Ministry of Education, Culture, Sports, Science and Technology, http:// www.mext.go.jp/

10. Fitzpatric SE, Reddy S, Lommel TS, Fischer JG, Speer EM, Stephene H, Park S, Johnson MA (2008) Physical activity and physical function improved following a community-based intervention in older adults in Georgia senior center. J Nutr Elder 27(1-2):135-154.

11. Petersen CB, Severin M, Hansen AW, Curtis T, Grønbæk M, et al. (2012) A population-based randomized controlled trial of the effect of combining a pedometer with an intervention toolkit on physical activity 
Citation: Maruo SJ, Sanongdetch W, Choeychom S, Chansirikarn S, Thrakul S, et al. (2015) Comparing the Effectiveness of Health Program in Thailand and Japan. J Nurs Care 4: 298. doi:10.4172/2167-1168.1000298

Page 6 of 6

among individuals with low levels of physical activity or fitness. Prev Med 54: 125-130.

12. Pelssers J, Delecluse C, Opdenacker J, Kennis E, Van Roie E, et al. (2013) "Every step counts!": effects of a structured walking intervention in a community-based senior organization. J Aging Phys Act 21: 167-185.

13. American College of Sports Medicine (2006) ACSM's guidelines for exercise testing and prescription 7th edition, Lippincott Williams \&Wilkins, Philadelphia.

14. Fuster J.M. The prefrontal cortex. Raven Press, New York. 1989.

15. Sasaki K, Nambu A, Tsujimoto T, Matsuzaki R, Kyuhou S, Gemba, H (1996) Studies on integrative functions of the human frontal association cortex with MEG. Cogn Brain Res 5: 165-74.

16. Goldman PS, Rosvold HE, Mishkin M (1970) Selective sparing of function following prefrontal lobectomy in infant monkeys. Exp Neurol 29: 221-226.

17. Nakade K, Abe K, Fujiwara T, Terasawa K, Okuhara M, Nakajima $K$, Yanagisawa A, Shinohara K, Matsumura T, Fujimori S, Miura H (2009) The influence of two different health education program on GO/NO-GO tasks, physical fitness tests and blood tests, Japan Society of Physical Anthropology 14,3,pp143-150.

18. Terasawa K, Matsumura T, Nakade K, Shinohara K, Fujimori S, Sasamori F, Terasawa S., Miura. H., Yanagisawa. A., Nakajima. K., Okuhara. M., Kobayashi. T., Zhang. Y., Hirota N, Amaiwa S, Katayama S (2008) Effects of walking on physical function, blood and GO/NO-GO tasks, poster session presented at the Asia-Pacific Conference on Mind Brain and Education.

19. Terasawa Koji, Wakimoto Koichi, Yoneyama Kenjirou, Terasawa Saiki, Kinoshita Shinya (2010) The relationship between the frontal lobe and physical strength.The 16th Biennial Conference,Preparing for the Aging Tsunami; Hawaii Pacific Gerontological Society,pp 23, Hawaii.

20. Mikawa K, Yano Y, Senjyu H (2012) Development of a field test for evaluating aerobic fitness. Int J Sports Med 33: 346-350.

21. Yokoya T, Demura S, Sato S (2009) Three-year follow-up of the fall risk and physical function characteristics of the elderly participating in a community exercise class. J Physiol Anthropol 28: 55-62. 